\title{
Glucocorticoids and neonatal brain injury: the hedgehog connection
}

\author{
Alberto Gulino, ${ }^{1,2}$ Enrico De Smaele, ${ }^{1}$ and Elisabetta Ferretti ${ }^{1}$ \\ ${ }^{1}$ Department of Experimental Medicine, Sapienza University, Rome, Italy. ${ }^{2}$ Neuromed Institute, Pozzilli, Italy.
}

\begin{abstract}
Glucocorticoids (GCs) play a critical role in neural development; however, their prenatal or neonatal therapeutic use can have detrimental effects on the developing brain. In this issue of the JCI, Heine and Rowitch report that the molecular mechanisms underlying these detrimental effects involve the sonic hedgehog (Shh) signaling pathway, a crucial regulator of brain development and neural stem/progenitor cells (see the related study beginning on page 267). They show that GCs suppress Shh-induced proliferation of cerebellar progenitor cells in postnatal mice and that, conversely, Shh signaling is protective against GC-induced neonatal cerebellar injury by inducing the enzyme 11 $\beta$ HSD2, which inactivates the GCs corticosterone and prednisolone, but not dexamethasone. The data provide a rationale for the therapeutic use of $11 \beta$ HSD2-sensitive GCs, but not dexamethasone, or for the exploitation of the neuroprotective effect of Shh agonists to prevent GCinduced pre- or neonatal brain injury.
\end{abstract}

\section{Corticosteroids and the brain}

The corticosteroid glucocorticoids (GCs), together with the sex steroids estrogen, progesterone, and testosterone, belong to the large family of steroid hormones. GCs are secreted by the adrenal cortex and exert their effects in target cells via binding to specific intracellular receptors (e.g., mineralocorticoid receptor and GC receptor [GR]) that activate or repress target gene transcription, although nongenomic signaling activity has also been reported (1). GRs mediate the effects of high levels of endogenous GCs, such as corticosterone (Cort) and cortisol, and also bind synthetic steroids, such as prednisolone (Pred), dexamethasone (Dex), or $\beta$-methasone. GCs exert a wide spectrum of influences on developing organs, including lung and brain. The brain sensitivity to GCs begins in embryonic life, as GR is expressed in fetal neurons (2) and is maintained in several adult brain regions, including the

Conflict of interest: The authors have declared that no conflict of interest exists.

Nonstandard abbreviations used: CGNP, cerebellar granule neuron precursor; Cort, corticosterone; COUP-TFII, chicken ovalbumin upstream promotertranscription factor II; Dex, dexamethasone; Dhh, desert hedgehog; GC, glucocorticoid; Gli, gliomaassociated oncogene; GR, GC receptor; Hh, hedgehog; $11 \beta$ HSD, 11 $\beta$-hydroxysterol dehydrogenase; Ihh, indian hedgehog; Pred, prednisolone; Ptch, Patched; Shh, sonic hedgehog; Smo, Smoothened.

Citation for this article: J. Clin. Invest. 119:243-246 (2009). doi:10.1172/JCI38387. hippocampus and cerebellar cortex (3). Exposure to supraphysiologic GC levels during embryonic life leads to alterations in neuronal proliferation, growth, and differentiation. In animal models, perinatal GC administration inhibits brain neuron division and reduces cerebellum and brain size and cell number, but may also cause hippocampal neuron death (2).

In the adult, GC excess may suppress neurogenesis in the subgranular layer of the dentate gyrus, a brain stem cell district in which new granular neurons are generated (4). Accordingly, adrenalectomy greatly increases neurogenesis in the dentate gyrus (5). It should be noted that GCs can also protect against neurodegeneration (5), suggesting that GCs are capable of exerting adaptive effects that prevent neural injury caused by overaggressive cellular defense mechanisms.

\section{Neural side effects of prenatal or} postnatal therapy with synthetic GCs A variety of synthetic GCs, including Pred, Dex, $\beta$-methasone, and hydrocortisone, have been created for therapeutic use, and the therapeutic benefits versus adverse effects of each must be considered when choosing which GC to administer. Prenatal treatment with synthetic GCs is commonly used for women at risk of preterm birth in order to prevent respiratory distress syndrome and neonatal death caused by inadequate surfactant production in fetal lungs (6).
However, this therapy has potential adverse effects on neurodevelopment (7). Postnatal GC therapy is used in the prevention and/or treatment of chronic lung disease in preterm babies. This therapy is effective in improving lung function and reducing neonatal mortality but is associated with long-term side effects, including altered neurodevelopment with increased risk of cerebral palsy and neurosensory disability (8). Delaying GC therapy could reduce, but not eliminate, the incidence of GC-mediated adverse neurological outcomes (9).

\section{Molecular mechanisms underlying GC-induced neurodevelopmental} injury: role of the hedgehog pathway Previously, the molecular mechanisms underlying GC-induced brain injury have been unclear. Heine and Rowitch now report, for what is believed to be the first time, the identification of antagonistic crosstalk between GCs and the sonic hedgehog (Shh) signaling pathway in proliferating cerebellar granule neuron precursors (CGNPs) in postnatal mice (10).

The hedgehog $(\mathrm{Hh})$ signaling pathway is crucial for the regulation of developmental processes and is activated by the binding of the secreted Hh proteins Shh, desert hedgehog (Dhh), and indian hedgehog (Ihh) to the transmembrane receptor Patched (Ptch) in target cells. Secreted Hh proteins, upon binding to Ptch, abolish a repression effect on a second transmembrane protein, Smoothened (Smo), which activates the downstream signaling cascade and the glioma-associated oncogene (Gli) family of transcription factors that regulate a number of target genes controlling cell proliferation and differentiation, such as those encoding $\mathrm{N}$-myc and D-type cyclins (11).

Hh signaling plays a paradigmatic role during cerebellar development, during which this pathway is a master promoter of the expansion of the CGNP population; it also plays a critical role in whole-brain morphogenesis (reviewed in refs. 12, 13). A specific emerging function of $\mathrm{Hh}$ signaling is the maintenance and self renewal of neu- 


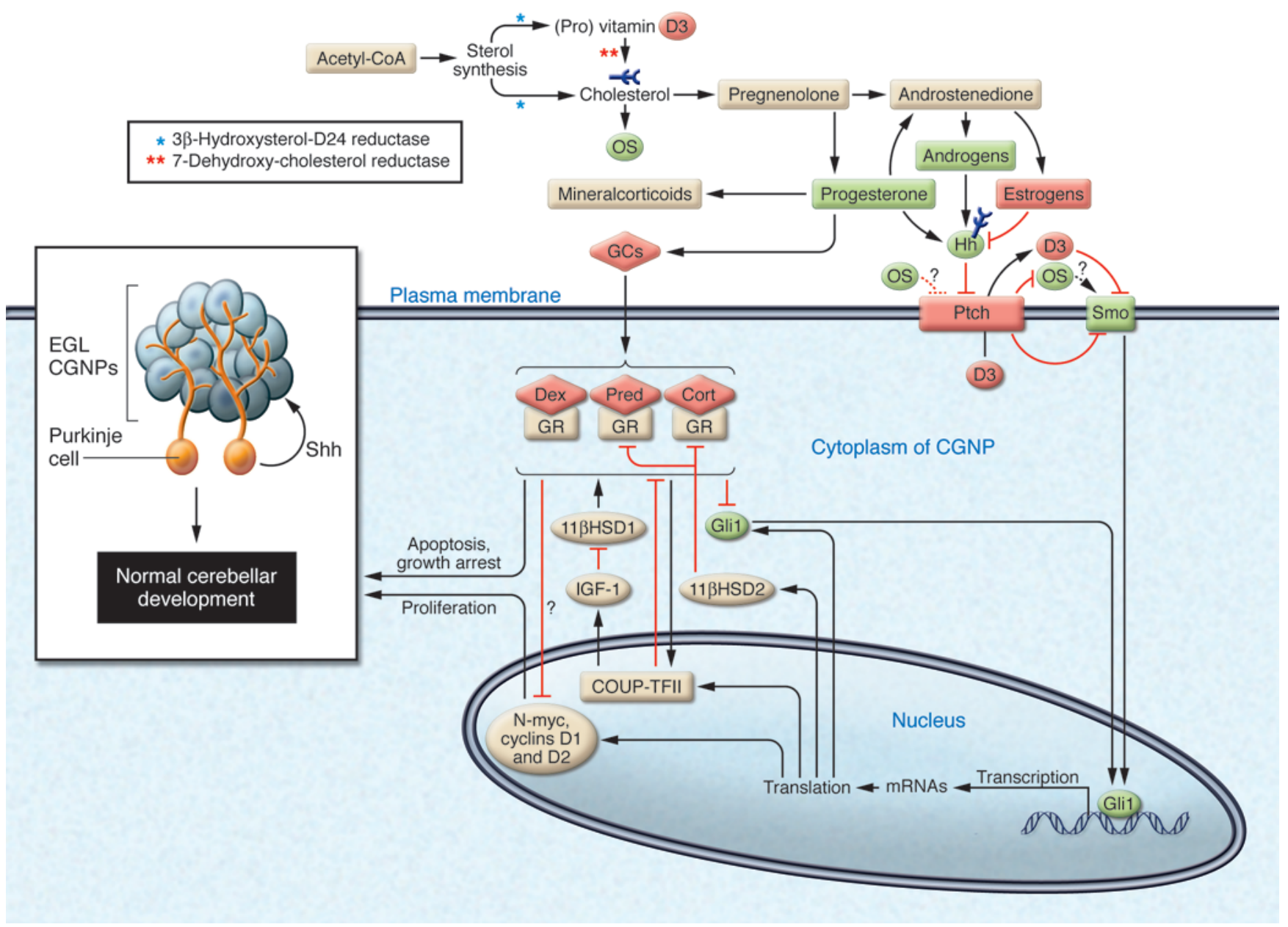

\section{Figure 1}

Crosstalk between $\mathrm{Hh}$ and steroid hormones during neuronal development and injury. Steroid hormone synthesis starts with acetyl-CoA and leads to the formation of (pro) vitamin D3 and cholesterol. Cholesterol-derived pregnenolone is synthesized from and is the origin of all steroid hormones. Cholesterol also gives rise to oxysterols (OS), important positive mediators of Hh pathway activation. Inhibition or mutation of enzymes involved at early steps of steroid hormone biosynthesis (asterisks) blocks cholesterol and (pro) vitamin D3 production, leading to defects in Hh signaling. Progesterone induces Ihh in the uterus, androgens upregulate Shh in the developing prostate, and estrogens downregulate Shh in the prostate and Ihh and Dhh in the uterus. In this issue of the $\mathrm{JCl}$, Heine and Rowitch report that GCs can modulate the Hh pathway in postnatal mice (10). Proliferation of CGNPs in the cerebellar external granule layer (EGL) is physiologically promoted by Shh secreted by Purkinje cells. This process is antagonized by binding of GCs to the GR, which also leads to CGNP apoptosis. GC antagonism of Hh signaling leads to neuronal injury and aberrant cerebellar development. Shh protects CGNPs via 11ßHSD2, which inactivates cortisol, Cort, and Pred, but not Dex, suggesting that it is better to use $11 \beta \mathrm{HSD} 2$-sensitive GCs to avoid neuronal injury in neonates. GCs may exert their effects via interaction with COUP-TFII: the GC-bound GR stimulates COUP-TFII-induced transactivation, while COUP-TFII represses GR transcriptional activity (27). COUP-TFII is also an Hh target and modulates the expression of IGF-1, which regulates GC metabolism through 11ßHSD1. Positive and negative regulators of the Hh pathway are shown in green and red, respectively.

ral progenitors in stem cell niches located in several regions of the embryonic, postnatal, and adult brain, thereby sustaining developmental and tissue repair processes (13).

Using mouse models, Heine and Rowitch show that chronic GC treatment of mouse pups from P0 through P7 inhibits cerebellar growth and proliferation of external granule layer CGNPs by promoting cell cycle exit and premature differentiation, whereas acute GC administration at P7 causes transient CGNP apoptosis (Figure 1 and ref. 10).
While they did not observe substantial modulation of GR expression or $S h b$ and Gli1 mRNA levels in vivo (albeit evaluated by nonquantitative immunohistochemical or in situ hybridization assays), in vitro analysis showed that the GC-induced antiproliferative effect on CGNPs may be caused by counteraction of the Shh-mediated proliferative pathway. Indeed, the authors observed a GC-driven reduction in protein levels of Shh targets Gli1, N-myc, and D-type cyclins in CGNPs (Figure 1).
The mechanism of action of GCs on these proteins was not identified, although GCs did not appear to act by modulating the mRNA levels of these target proteins. Nevertheless, it seems reasonable that modulation of Gli1 protein translation or stability would be the initial event, although a direct effect on N-myc and D-type cyclins cannot be ruled out. More importantly, the authors have unveiled the existence of a crossantagonism between GCs and Shh signaling, based on their finding that both Shh 
treatment in vitro and overexpression of the constitutively activated form of Smo in vivo counteract the antiproliferative effects of GC treatment in CGNP cultures (10).

The authors also investigated the mechanism through which Shh may antagonize GCs (10). Both $11 \beta$-hydroxysterol dehydro-

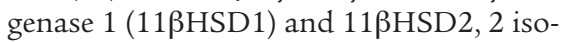
zymes of $11 \beta \mathrm{HSD}$, catalyze the conversion of hormonally active GCs to inactive cortisone, and vice versa. While $11 \beta \mathrm{HSD} 2$ converts endogenous GCs (e.g., Cort and cortisol) and some synthetic GCs (e.g., Pred, but not Dex) into inactive 11-ketoderivatives (cortisone), 11ßHSD1 mainly converts cortisone to active GCs (14).

The authors show that Shh signaling potently induces $11 \beta$ HSD2 upregulation and by this mechanism reduces the activity of GCs in CGNPs (Figure 1 and ref. 10). The protective effects exerted by Shh through $11 \beta$ HSD2 upregulation were further documented by the increase in GC toxicity on CGNPs following administration of the 11ßHSD2 inhibitor carbenoxolone.

In summary, the findings described in the current study reveal another important mechanism of action of the Hh pathway during the critical phases of cerebellar development and most likely other organ morphogenesis, improving our consciousness of the complex interplay between hormones and developmental pathways.

\section{Crosstalk between steroids and the Hh pathway}

The findings of Heine and Rowitch (10) add another piece of information regarding the importance, for proper Hh pathway function and regulation, of steroid hormones and their biosynthetic pathway, which includes intermediates such as (pro) vitamin D3, cholesterol, and its derivatives, the oxysterols. Covalent binding of cholesterol at the C-terminal domain of Hh protein is required for proper Hh extracellular diffusion (15), and in animal models, disruption of cholesterol synthesis processes leads to developmental malformation (e.g., holoprosencephaly) that mimics malformations observed in Hh mutant mice (15).

Hh signaling is instead regulated by cholesterol derivatives. For instance, oxysterols may activate the Smo receptor via direct interaction (16), or indirectly by binding to Ptch and inhibiting its activity (17), while (pro) vitamin D3, which is secreted through Ptch, can bind to and inhibit Smo (18) (Figure 1). Steroid hormones have previously been described as being involved in interplay with the Hh signaling pathway, either as regulators of the pathway or as targets. Ihh is positively modulated by progesterone in the uterus during progesterone-induced processes of proliferation, angiogenesis, and decidualization, which are critical for embryo implantation (19). In contrast, Ihh and Dhh are downregulated by estrogens in the uterus (20). Finally, estrogens and androgens have previously been implicated in the negative and positive regulation, respectively, of Shh expression during prostate gland development $(21,22)$. Therefore, the Hh pathway is emerging as an important mediator of the effects of steroid hormones (Figure 1).

In contrast, the reverse ability of Hh to control steroid hormone function is still poorly understood. In addition to the candidates described in the current report (10), the Hh target chicken ovalbumin upstream promoter-transcription factor II (COUP-TFII; ref. 23), a member of the nuclear orphan receptor superfamily, is a good candidate for GC control (Figure 1). COUP-TFII plays a critical role in the development of CNS and cerebellum (24) and may modulate GCs through IGF-1 and 11ßHSD1 (Figure 1 and refs. 24, 25).

\section{Clinical relevance and perspectives}

Heine and Rowitch have shed light on the complex interplay existing between steroid hormones and pathways involved in development and cell stemness (10). Their observations regarding the cross-regulatory mechanisms at play between $\mathrm{Hh}$ and GCs raise new questions and have several molecular and clinical implications.

From the molecular point of view, 2 open questions remain, regarding (a) the mechanism through which GCs modulate Hh target genes and (b) how Hh is able to upregulate 11ßHSD2. Knowledge of the underlying molecular events in detail will allow the development of new molecules and more targeted treatments, which could render therapeutic intervention with GCs more effective and less neurotoxic.

From the clinical point of view, the authors point out that $11 \beta \mathrm{HSD} 2$, which inactivates a subset of steroid hormones (e.g., Cort, hydrocortisone, and Pred), is not able to efficiently inactivate the synthetic GCs Dex or $\beta$-methasone; they propose a rationale for the use of $11 \beta$ HSD2-sensitive GCs instead of Dex in neonatal infants, especially in the most vulnerable, critical first postnatal week of life (10). Regarding the proposed use of small-molecule Hh agonists to activate the neuroprotective role of this pathway, caution should be used because of the well-documented role of $\mathrm{Hh}$ in cerebellar neuronal tumorigenesis $(12,13)$.

Finally, there are possible implications regarding mechanisms of neurogenesis and neurodegeneration, which have been shown to be modulated by both Hh signaling and GCs. New neurons developing from neural stem cells or multipotent progenitor cells populate the adult hippocampus throughout life and contribute to cognitive functions, including learning and memory (26). Discovery of natural molecular regulators by which GCs and Hh regulate each other during adult neurogenesis is important for the development of pharmacological drugs and of successful neuron replacement strategies to treat neurodegenerative diseases.

\section{Acknowledgments}

The authors are supported by Telethon Grant GGP07118, Associazione Italiana per la Ricerca sul Cancro, the Italian Ministry of University and Research, the Italian Ministry of Health, and the Pasteur Institute-Cenci Bolognetti Foundation. The authors thank Andrea Dotta for helpful comments.

Address correspondence to: Alberto Gulino, Department of Experimental Medicine, Sapienza University, 324 Viale Regina Elena, Roma, Italy. Phone: 39-064958637; Fax:39-064461974; E-mail: alberto.gulino@ uniroma1.it.

1. Stahn, C., and Buttgereit, F. 2008. Genomic and nongenomic effects of glucocorticoids. Nat. Clin. Pract. Rheumatol. 4:525-533.

2. McEwen, B.S. 1987. Steroid hormones and brain development: some guidelines for understanding actions of pseudohormones and other toxic agents. Environ. Health Perspect. 74:177-184.

3. Ozawa, H. 2005. Steroid hormones, their receptors and neuroendocrine system. J. Nippon Med. Sch. 72:316-325.

4. McEwen, B.S. 2005. Glucocorticoids, depression, and mood disorders: structural remodeling in the brain. Metabolism. 54:20-23.

5. Nichols, N.R., Agolley, D., Zieba, M., and Bye, N. 2005. Glucocorticoid regulation of glial responses during hippocampal neurodegeneration and regeneration. Brain Res. Brain Res. Rev. 48:287-301.

6. Miracle, X., Di Renzo, G.C., Stark, A., Fanaroff, A., Carbonell-Estrany, X., and Saling, E. 2008. Guideline for the use of antenatal corticosteroids for fetal maturation. J. Perinat. Med. 36:191-196.

7. Crowther, C.A., Doyle, L.W., Haslam, R.R., Hiller, J.E., Harding, J.E., and Robinson, J.S. 2007. Outcomes at 2 years of age after repeat doses of antenatal corticosteroids. N. Engl.J. Med. 357:1179-1189.

8. Halliday, H.L., Ehrenkranz, R.A., and Doyle, L.W. 2003. Early postnatal ( $<96$ hours) corticosteroids for preventing chronic lung disease in preterm infants. Cochrane Database Syst. Rev. CD001146.

9. Halliday, H.L., Ehrenkranz, R.A., and Doyle, L.W. 
2003. Delayed (>3 weeks) postnatal corticosteroids for chronic lung disease in preterm infants. Cochrane Database Syst. Rev. CD001145.

10. Heine, V.M., and Rowitch, D.H. 2009. Hedgehog signaling has a protective effect in glucocorticoidinduced mouse neonatal brain injury through an $11 \beta$ HSD2-dependent mechanism. J. Clin. Invest. 119:267-277

11. Varjosalo, M., and Taipale, J. 2008. Hedgehog: functions and mechanisms. Genes Dev. 22:2454-2472.

12. Gulino, A., Di Marcotullio, L., Ferretti, E., De Smaele, E., and Screpanti, I. 2007. Hedgehog signaling pathway in neural development and disease. Psychoneuroendocrinology 32(Suppl 1):S52-S56.

13. Ruiz i Altaba, A. 2006. Hedgehog-Gli signaling in buman disease. Landes Bioscience. Georgetown, Texas, USA. 228 pp.

14. Holmes, M.C., and Seckl, J.R. 2006. The role of 11 beta-hydroxysteroid dehydrogenases in the brain. Mol. Cell. Endocrinol. 248:9-14.

15. Lewis, P.M., et al. 2001. Cholesterol modification of sonic hedgehog is required for long-range signaling activity and effective modulation of signaling by Ptc1. Cell. 105:599-612.
16. Corcoran, R.B., and Scott, M.P. 2006. Oxysterols stimulate Sonic hedgehog signal transduction and proliferation of medulloblastoma cells. Proc. Natl. Acad. Sci. U. S. A. 103:8408-8413.

17. Dwyer, J.R., et al. 2007. Oxysterols are novel activators of the hedgehog signaling pathway in pluripotent mesenchymal cells. J. Biol. Chem. 282:8959-8968.

18. Bijlsma, M.F., et al. 2006. Repression of smoothened by patched-dependent (pro-)vitamin D3 secretion. PLoS Biol. 4:e232.

19. Lee, K., et al. 2006. Molecular mechanisms involved in progesterone receptor regulation of uterine function. J. Steroid Biochem. Mol. Biol. 102:41-50

20. Katayama, S., et al. 2006. The expression of Hedgehog genes (Ihh, Dhh) and Hedgehog target genes (Ptc1, Gli1, Coup-TfII) is affected by estrogenic stimuli in the uterus of immature female rats. Toxicol. Appl. Pharmacol. 217:375-383.

21. Prins, G.S., Huang, L., Birch, L., and Pu, Y. 2006 The role of estrogens in normal and abnormal development of the prostate gland. Ann. N. Y. Acad. Sci. 1089:1-13.

22. Pu, Y., Huang, L., Birch, L., and Prins, G.S. 2007.
Androgen regulation of prostate morphoregulatory gene expression: Fof10-dependent and -independent pathways. Endocrinology. 148:1697-1706.

23. Krishnan, V., et al. 1997. Mediation of Sonic hedgehog-induced expression of COUP-TFII by a protein phosphatase. Science. 278:1947-1950.

24. Kim, B.J., Takamoto, N., Yan, J., Tsai, S.Y., and Tsai, M.J. 2008. Chicken ovalbumin upstream promotertranscription factor II (COUP-TFII) regulates growth and patterning of the postnatal mouse cerebellum. Dev. Biol. Online publication ahead of print. doi:10.1016/j.ydbio.2008.11.001.

25. Agha, A., and Monson, J.P. 2007. Modulation of glucocorticoid metabolism by the growth hormone - IGF-1 axis. Clin. Endocrinol. (Oxf). 66:459-465.

26. Hagg, T. 2007. Endogenous regulators of adult CNS neurogenesis. Curr. Pharm. Des. 13:1829-1840.

27. De Martino, M.U., Alesci, S., Chrousos, G.P., and Kino, T. 2004. Interaction of the glucocorticoid receptor and the chicken ovalbumin upstream promoter-transcription factor II (COUP-TFII): implications for the actions of glucocorticoids on glucose, lipoprotein, and xenobiotic metabolism. Ann. N. Y. Acad. Sci. 1024:72-85.

\title{
DAMPs ramp up drug toxicity
}

\author{
Jacquelyn J. Maher
}

Department of Medicine and Liver Center, UCSF, San Francisco, California, USA.

\begin{abstract}
The clinical syndrome of acetaminophen-induced liver injury represents the combined result of drug toxicity and a potent innate immune response that follows drug-induced cell death. In this issue of the JCI, Imaeda and colleagues report that DNA released from dying hepatocytes is a key stimulus of innate immune activation in the acetaminophen-treated mouse liver (see the related article beginning on page 305 ). They present evidence indicating that hepatocyte DNA promotes immune activation by acting as a danger-associated molecular pattern (DAMP) that stimulates cytokine production in neighboring sinusoidal endothelial cells via Tlr9 and the Nalp3 inflammasome.
\end{abstract}

The analgesic acetaminophen is widely known for its potential to cause severe and sometimes lethal liver injury. When ingested in large amounts, acetaminophen overwhelms the normal metabolic pathways of glucuronidation and sulfation and undergoes oxidation to form the highly reactive intermediate $N$-acetyl-p-benzoquinone-imine (NAPQI). NAPQI is not harmful if it combines rapidly with glutathione; however, when hepatic glutathione stores

Conflict of interest: The author has declared that no conflict of interest exists.

Nonstandard abbreviations used: DAMP, dangerassociated molecular pattern; HMGB1, high-mobility group box 1; NALP3, NACHT, LRR, and pyrin domain-containing protein 3; NAPQI, $N$-acetyl- $p$-benzoquinone-imine; NLR, nucleotide-binding domain, leucine-rich repeat-containing protein; SEC, sinusoidal endothelial cell.

Citation for this article: J. Clin. Invest. 119:246-249 (2009). doi:10.1172/JCI38178. are depleted, NAPQI escapes detoxification, resulting in liver cell death (1). An important but underappreciated aspect of acetaminophen toxicity is that direct, drug-induced harm accounts for only part of the overall syndrome of acetaminophen-induced liver injury. The reason for this is that the initial wave of drug-induced hepatocellular destruction is followed by a robust innate immune response, in which invading inflammatory cells release toxic oxidants and cause a second wave of destruction. The collateral damage inflicted by inflammatory cells can be so severe as to double the degree of tissue injury caused by acetaminophen alone (2).

\section{Innate immunity is the result of danger signaling}

Activation of the innate immune system following a noninfectious insult such as drug toxicity arises when dying cells release molecules that serve as "danger signals." Danger molecules trigger inflammation by engaging pattern recognition receptors such as TLRs (3) and nucleotide-binding domain, leucine-rich repeat-containing proteins (NLRs) (4) and are thus referred to as danger-associated molecular patterns (DAMPs) (5). Through TLRs, DAMPs signal cytokine and chemokine production and upregulate the expression of celladhesion molecules. When DAMPs interact with NLRs, they stimulate NLRs to complex with ASC (apoptosis-associated speck-like protein containing a CARD) to form macromolecular complexes called inflammasomes, which activate caspase-1 and stimulate cleavage of the proinflammatory cytokines pro-IL- $1 \beta$ and pro-IL-18 to their mature forms, IL-1 $\beta$ and IL-18 (6). Self molecules that act as DAMPs interact primarily with TLR2, -4 , and -9 and an NLR with an $\mathrm{N}$-terminal pyrin domain designated NACHT, LRR, and pyrin domain-containing protein 3 (NALP3; also known as NLRP3). The list of these molecules is rapidly growing (Table 1), emphasizing the importance of endogenous danger signaling to a broad array of medical disorders ranging from gout to systemic lupus erythematosus to Alzheimer disease (7-9). A danger molecule that is believed to play a 